Relations industrielles

Industrial Relations

\title{
Le 2e Congrès de la FTQ (Québec, 1958)
}

\section{Guy Lamarche}

Volume 14, numéro 1, janvier 1959

URI : https://id.erudit.org/iderudit/1022337ar

DOI : https://doi.org/10.7202/1022337ar

Aller au sommaire du numéro

\section{Éditeur(s)}

Département des relations industrielles de l’Université Laval

\section{ISSN}

0034-379X (imprimé)

1703-8138 (numérique)

Découvrir la revue

\section{Citer ce document}

Lamarche, G. (1959). Le 2e Congrès de la FTQ (Québec, 1958). Relations industrielles / Industrial Relations, 14(1), 51-58.

https://doi.org/10.7202/1022337ar
Résumé de l'article

En novembre dernier, la Fédération des travailleurs du Québec (CTC) a tenu son second congrès annuel. L'auteur tente de dégager les grandes lignes de forces qui se sont manifestées en cette circonstance.
Tous droits réservés @ Département des relations industrielles de l’Université Laval, 1959
Ce document est protégé par la loi sur le droit d'auteur. L’utilisation des services d'Érudit (y compris la reproduction) est assujettie à sa politique d'utilisation que vous pouvez consulter en ligne.

https://apropos.erudit.org/fr/usagers/politique-dutilisation/ 


\section{COMMENTAIRES}

\section{Le 2e Congrès de la FTQ (Québec, 1958)}

\section{Gư Lamakcife}

En novembre dernier, la Fédération des travailleurs du Québec (CTC) a tenu son second congrès annuel. L'auteur tente de dégager les grandes lignes de forces qui se sont manifestées en cette circonstance.

Les congrès annuels de la Confédération des travailleurs catholiques du Canada et de la Fédération des travailleurs du Québec (CTC) sont, dans la province de Québec, les deux événements syndicaux les plus attendus par les observateurs, parce qu'ils permettent assez bien de prendre la mesure des deux groupements, de constater leur évolution ou leur recul, d'apprécier leur dynamisme ou leur nonchalance. Dans la mesure où on peut percevoir le sens exact des résolutions et déceler les motifs d'action des chefs syndicalistes, un congrès est l'occasion rêvée pour faire subir à nos groupements syndicaux un examen général. C'est aussi une occasion redoutable de commettre des erreurs de diagnostic.

Les congrès ont en effet ce caractère de "préméditation 》 qui permet aux dirigeants d'en faire soit des mises en scène montées avec raffinement, soit des occasions pour le mouvement ouvrier de crier publiquement ses aspirations. Tout dépend de la «surveillance démocratique » et du climat dans lequel elle peut s'exercer. Entre deux extrêmes dont l'une est l'utilisation d'un congrès pour bâtir son prestige et l'autre l'utilisation de son prestige pour «bâtir» un congrès, il y a tous les degrés de la démocratie, depuis celle qui existe dans une constitution jusqu'à celle qui vit dans la réalité.

Il ne s'agit pas dans ce préambule d'indiquer à quelle sorte de démocratie loge la FTQ, ni d'évaluer les richesses de ses structures démocratiques, encore moins d'établir une comparaison avec d'autres centrales syndicales. Mais il apparait important avant de traiter d'un congrès en particulier - celui de Québec en novembre 1958 - de donner quelques indications sur la «personnalité » de la FTQ.

Dans un article publié récemment dans cette même revue, l'abbé Gérard Dion a touché du doigt une des caractéristiques fondamentales de la FTQ, quand il s'est référé à ses structures constitutionnelles pour 
en expliquer le caractère gélatineux, les équivoques et le manque d'homogénéité qui résultent de son peu d'infiuence sur ses syndicats affiliés.

La FTQ fait difficilement bouger les gros syndicats; on peut même dire que c'est le contraire. On ne saurait donc juger la Fédération sans d'abord évaluer dans l'action ses principaux syndicats affiliés, ce qui implique qu'on les a suivis pendant un certain nombre d'années et qu'on en connaît les dirigeants au moins autant qu'on connaît les officiers élus de la FTQ. Ces derniers ont certes un grand prestige, mais ils nont pas d'autorité directe sur les syndicats, ou s'ils en ont, c'est qu'ils sont en même temps dirigeants de syndicats importants.

Il faut s'empresser d'ajouter que malgré cette faiblesse, la FTQ a acquis depuis trois ans un dynamisme réel, souvent en retard sur les événements, mais qui fut heureusement alimenté par quelques hommes remarquables. Au moment de sa fondation à Québec en 1956, on a pu craindre avec raison que les meilleurs éléments de l'ancienne FUIQ, tenants d'un syndicalisme combatif et partisans de l'action sur tout les plans, ne soient à jamais noyés dans une masse assez peu vigoureuse qui formait avant la fusion la Fédération du travail du Québec (FAT). Le feu sacré au lieu de s'éteindre a gagné du terrain et iI faut signaler certaines «conversions», si étonnantes qu'on s'en méfie encore. Si le syndicalisme d'affaires prévaut encore dans trop de syndicats, il a perdu sa primauté à la Fédération elle-même.

Souligons enfin que la FTQ reste un des mouvement les plus démocratiques de la province. Sa démocratie fut à quelques occasions viciée par un byzantinisme regrettable, elle fut souvent passive, mais elle fut.

Ceci dit pour permettre au lecteur de prendre une attitude avertie en face de la FTQ, tentons d'examiner les lignes de force du dernier congrès.

\section{LES TEXTES OFFICIELS}

Parler du dernier congrès est évidemment s'engager à ne parler pratiquement que d'action politique. Nous en parlerons en deux étapes: d'abord à partir de textes officiels qui décrivent les buts et méthodes de la FTQ, et ensuite à partir de l'observation empirique. L'utilisation des textes ayant le désavantage de désincarner l'objet de notre étude, les observations personnelles qui suivront permettront de serrer de plus près la réalité de la FTQ. Du moins nous l'espérons.

Pour celui qui lit la constitution et la déclaration de principes de la FTQ, le troisième congrès s'est conformé en tous points à ces deux 
documents quand il adopta une résolution priant son exécutif d'entreprendre des pourparlers avec d'autres groupes en vue de la formation d'un parti politique provincial. On sera étonné peut-être de constater que tout cela était en puissance dans les textes alors que l'on croyait la FTQ très peu préoccupée d'action politique jusqu'à son dernier congrès. C'est peut-être qu'il ne faut pas donner trop d'importance aux constitutions et aux merveilleuses déclarations sur le papier.

On lit dans la Constitution de fusion, adoptée en 1956, que la FTQ s'est fixé les buts suivants:

1-De supporter les principes et la politique du Congrès du travail du Canada;

2-De promouvoir les intérêts de ses organisations affiliées et généralement d'aider à l'avancement du bien être social et économique des travailleurs du Québec;

5-D'obtenir la législation provinciale qui sauvegardera et promouvera les conventions de travail libres, les droits des travailleurs et le bien-être de la population en général.

6-De protéger et de renforcer nos institutions démocratiques, d'obtenir la reconnaissance et la pleine jouissance des droits et libertés qui nous reviennent en toute justice, et de préserver et perpétuer les traditions chères à notre démocratie.

10-Tout en protégeant l'indépendance du mouvement ouvrier de toute sujétion politique, d'encourager les travailleurs à voter, à exercer tous leurs droits et à prendre toutes les responsabilités de citoyens, et à faire leur juste part dans la vie politique des gouvernements fédéral, provincial et municipal.

On pourrait gloser longtemps sur la façon dont ces articles ont été ou plus exactement n'ont pas été appliqués jusqu'à maintenant. On peut tout de suite en tirer les conclusions suivantes.

Le premier but qui est de «supporter (entendre appuyer) la politique du CTC » reçoit évidemment une application directe et totale par la récente résolution politique de la FTQ. Nous en parlerons plus longuement tout à l'heure.

Quant aux autres articles qui ont trait à la protection des intérêts, des droits et des libertés démocratiques, à l'éducation et à l'action politique des travailleurs syndiqués, ils prennent dans la résolution de Québec, une dimension nouvelle, une actualité brûlante, alors même qu'on se demande si on les avait relus depuis le congrès de fusion. La décision de fonder un nouveau parti politique dépasse évidemment ce que contiennent ces articles, même implicitement. C'est-à-dire que 
pour défendre les intérêts des travailleurs, la FTQ en est venue à la conclusion que ses méthodes clasiques ne suffisent pas.

Mais pour arriver à cette conclusion, c'est plutôt à la déclaration de principes qu'il faut se référer.

\section{LA DÉClaRATION DE PRINCIPES}

Il convient de savoir que la Fédération des travailleurs du Québec possède depuis plus d'un an une fort belle déclaration de principes. Son adoption a été, il est vrai, plutôt silencieuse parce que le congrès qui en adopta une petite partie référa tout le reste au Conseil exécutif et aussi parce que les positions de principes à la FTQ n'ont jamais pris une grande importance aux yeux des observateurs qui ont été habitués à un certain opportunisme et à une prudente absence de toute idéologie qui dépasserait les principes de base du syndicalisme. Le congrès qui adopta la déclaration de principe à Montréal, en novembre 1957, attira plutôt l'attention par sa campagne électorale intra muros.

C'est pour cela que personne vraiment n’a été ému de voir que la FTQ devenait sur le papier partisane de l'éducation gratuite à tous les niveaux et d'un système complet de sécurité sociale, anti-capitaliste et favorable à l'étatisation pour ce qui regarde les ressources naturelles, tenante d'une éducation et d'une action politiques énergiques. Sur ce dernier chapître surtout on pourrait s'étonner que la même personne ait invité les syndicats à s'en tenir à une éducation politique éclairée pour présider un mois plus tard à une assemblée du Conseil exécutif qui adoptait le texte suivant:

«Les rudes expériences d'un passé récent ont donné de la maturité à notre mouvement et l'ont rendu conscient du rôle politique qu'il doit jouer, dans le cadre de notre système démocratique parlementaire, aussi bien par le vote de ses membres que par les pressions collectives de ses divers organismes.

La Fédération des travailleurs du Québec a acquis la conviction que l'expansion du syndicalisme et l'avènement de la sécurité sociale, si nécessaire à la famille du travailleur, ne pourront jamais être assurés par les forces politiques en place. Aussi ne voit-elle de solution que dans l'éducation et l'action politiques, l'une ne pouvant aller sans l'autre.

Elle entend donc accélérer l'éducation politique de ses membres, étudier les structures politiques actuelles, y chercher les correctifs nécessaires, et, pourvoir à leur application, inciter ses membres à l'action politique directe.

A ce propos, la Fédération étudie les solutions qui s'offrent à elle, et se propose d'en inventer qui lui soient propres, au besoin. Elle se propose de réexaminer constamment la situation politique à la lumière des facteurs nouveaux qui peuvent se présenter à son attention, et de dresser ainsi un programme d'action directe à l'in- 
tention de ses membres. Dans l'immédiat, elle voit la nécessité de donner son appui au parti politique qui lui fournira les meilleures garanties en travaillant sérieusement et de façon conskante dans l'intérêt de la classe laborieuse.

La Fédération entend collaborer en outre avec tous les groupes de citoyens qui s'intéressent activement à la régénération de la démocratie politique sur le plan provincial... »

Voilà bien en toutes lettres l'annonce d'une action politique très prochaine. On s'étonne maintenant que le texte ait eu très peu de retentissement au moment de son adoption, mais c'est peut-être qu'on n'y croyait pas vraiment. Pour plusieurs c'était un charmant texte de plus. Il ressuscite aujourd'hui après avoir dormi pendant un an, ou plutôt après une année de gestation.

La question est de savoir si c'est le texte qui a été mis en application ou si c'est l'application qui, pour une fois, rejoint le texte. Nous croyons que si la décision politique avait dû attendre le seul cheminement des idées dans les esprits et les coeurs, elle serait encore loin. Les hommes ont rarement forcé les événements à la FTQ: il leur ont répondu. (Nous touchons ici une différence profonde avec la CTCC où les conceptions intellectuelles et les déductions de la logique servent souvent de motifs d'action, de principal moteur).

A notre avis, c'est par des pressions venues de l'extérieur surtout que la FTQ a été amenée à décider de fonder un parti politique sur le plan provincial. Ceci est très important, car la loi de l'inertie fera que si ces pressions diminuent ou disparaissent, s'en sera fait de laction d'envergure à laquelle vise la FTQ. A moins que les forces internes qui agissent déjà au sein de la FTQ n'aient pris alors une influence décisive. Voyons donc quelles sont ces forces internes qui ont poussé la FTQ à envisager ou du moins à hâter une action politique aussi directe et aussi concrète que la formation d'un parti politique «progressiste».

\section{INFLUENCES EXTÉRIEURES}

La cause la plus immédiate, la plus directe et la plus influente de l'adoption par la FTQ de sa résolution politique est évidemment l'adoption d'une résolution semblable quelques mois auparavant, à Winnipeg, par le Congrès du travail du Canada. Au printemps de 1958, le CTC a décidé d'entreprendre des pourparlers en vue de la formation d'un parti politique sur le plan national. Furent invités à se joindre à ce nouveau mouvement tous les groupements et citoyens à mentalité «libérale», désireux de voir arriver au pouvoir politique un parti du peuple, authentiquement démocratique et bâti autour des idées généreuses de justice sociale, de sécurité sociale, de plein emploi, etc. Le Parti social démocratique était invité au premier chef, en tant qu'élément essentiel devant être à la fois le noyau, le précurseur et un des éléments constituants du nouveau mouvement. 
Il est vrai que «le tout contient habituellement la partie», mais il n'était pas acquis d'avance que la FTQ appuierait cette politique nouvelle du CTC. Sa longue répugnance à organiser toute action politique unifiée et systématique, le peu d'enthousiasme qu'elle a tou. jours manifesté pour le PSD à l'exception d'une minorité, une sorte de nonchalance à inventer des formules d'action qui impliqueraient une grande dépense d'énergie, tout cela n'a pas préparer la FTQ à marcher à fond derrière le CTC. Pour plusieurs, la délégation de Québec s'était en grande partie laissé faire, à Winnipeg, préférant la non résistance à l'opposition ouverte.

C'est donc avec un certain étonnement que l'on a entendu à Québec des hommes qui étaient contre l'action politique à Winnipeg faire un assez vigoureux discours en faveur de la «politique du Congrès» à Québec. Il faut que soit bien forte cette discipline nationale, sorte de respect spontané envers la «politique du Congrès». Il faut aussi que ce soient opérées de profondes transformations au sein même de la FTQ, mais cela fera l'objet du prochain chapitre.

Le CTC possède donc maintenant une influence marquante sur ses syndicats affiliés, influence engendrée par une atmosphère qui empêche décemment un syndicat de condamner l'action de la majorité, même si, lui, il ne veut pas «marcher». Encore l'an dernier, de gros syndicats possédaient implicitement un droit de veto au CTC, en ce sens qu'on n'aurait pas oser entreprendre une action à laquelle ils auraient été irréductiblement opposée. Sur la question de la politique, on sent au contraire aujourd'hui que certains de ces syndicats et leurs dirigeants doivent se mettre au pas pour ne pas perdre leur prestige.

Le plus étonnant dans tout, cela, c'est que la FTQ est allée encore plus loin que ne l'exigeait son affiliation au CTC. Alors que le congrès de Québec aurait pu simplement se dire d'acord avec le projet de fonder un parti politique national, il a décidé carrément d'entreprendre des pourparlers avec les groupements progressistes et libéraux de cette province en vue de la création d'un nouveau parti provincial. Il fallait d'autres motifs que la résolution de Winnipeg pour que la FTQ concoive ainsi sa responsabilité sur le plan politique.

Le premier de ces motifs est que le Québec est devenu soudainement la province canadienne la plus féconde en groupements politiques nouveaux, tous d'opposition. Les uns s'attaquent au régime et prétendent, en le remplaçant, faire renaître un climat de liberté et de moralité publique. D’autres accusent principalement le système économique et social qui prévaut encore, système qui entraînerait nécessairement les hommes dans leurs fautes actuelles.

Il devient à la mode, sans doute pour des raisons très profondes que nous révéleraient les sciences politiques et la sociologie, de vouloir regrouper ces forces d'opposition contre l'ennemi commun. Les 
citoyens sont disposés à acclamer l'homme ou le groupe qui y réussira. La FTQ a décidé d'essayer, forte de l'exemple du CTC sur le plan national. Le Québec est de toute évidence à la veille de transformations politiques, et la FTQ désire que ce soit aussi des transformations économiques et sociales.

Ajoutons à cela que c'est dans le Québec que l'opposition patronale au syndicalisme a connu certains succès grâce surtout à une tolérance évidente - quand ce n'était pas une complicité - du gouvernement provincial. Ce genre de phénomène a toujours engendré un réveil chez ceux qui en ont souffert. En réagissant avec une détermination inaccoutumée, le syndicalisme est sorti vainqueur de la plupart de ses luttes économiques. Aussitôt est née la confiance qu'il pourra devenir tout aussi efficace contre la campagne d'opposition par une lutte politique.

Le gouvernement a ajouté à ce mouvement de défense (passer à l'attaque, c'est souvent défendre sa vie et sa liberté) un aiguillon particulier par la façon dont il a reçu les doléances de la FTQ quand celleci présenta son mémoire quelques semaines avant le congrès. Mais restons sereins.

Enfin, mentionnons comme autre motif extérieur les victoires des syndicats ouvriers aux élections américaines quelques jours seulement avant le congrès. Cela démontrait aux syndicats québecois qu'ils pouvaient être efficaces sur le plan politique, puisque d'autres syndicats l'avaient été ailleurs.

\section{FORCES INTERNES}

Il serait peu flatteur pour la FTQ et contraire à la vérité de dire que cette centrale syndicale n'a obéi qu'à des forces externes pour adopter sa résolution politique. D'ailleurs, le seul fait que ces forces aient pu agir indique une réceptivité chez la FTQ, une non-répugnance à accepter ce que les événements lui demandaient de faire. Combien d'idées généreuses seraient projetées dans l'action si les hommes ou les groupes qui les reçoivent avaient au moins la volonté d'y répondre. Une terre fertile est aussi importante que l'action du semeur pour qu'on puisse espérer quelque récolte.

Le seul fait qu'il n'était plus invraisemblable que la FTQ adopte une pareille résolution montre un remarquable progrès sur ce qu'était la FTQ au moment de la fusion en 1956, et même un an avant le congrès de Québec. Ce qui hier était considéré comme irréalisable, révolutionnaire, trop avancé, dangereux et non souhaitable, devient aujourd'hui impérieux, urgent, nécessaire et indispensable. L'idéaliste d'hier devient l'homme de vision d'aujourdhui, et celui qui hier était réaliste est aujourd'hui réactionnaire. Il est vrai que cette transforma- 
tion s'est accomplie en si peu de temps qu'un certain doute subsiste quand à la profondeur de cette révolution.

Mais ne serait-elle que verbale chez plusieurs, elle donne maintenant les coudées franches à ceux qui, au sein de la FTQ, ont toujours préconisé les idées qui gravitent autour de l'action politique. Cette minorité agissante voit aujourd'hui ses idées triompher. Son travail dans les syndicats, les comités, les conseils de travail et au sein de la Fédération y aura été pour quelque chose.

Une autre force qu'il ne faut pas négliger dans la FTQ est le besoin presque physique d'unité sur toutes les grandes questions. On pourrait presque parler d'un besoin sentimental d'unanimité. Sur ce problème, on peut dire que tous travaillent avec acharnement. Aux heures difficiles que va traverser encore le syndicalisme, et aux heures où le mouvement syndical portera une plus large responsabilité de l'avenir de notre société, il faudra qu'il soit très uni. On a vu le congrès de la FTQ souhaiter avec une ardeur absolument neuve l'affiliation de la CTCC au CTC. C'est parce qu'on sent bien qu'avec un mouvement syndical désuni, il est inutile de songer à des transformations politiques en profondeur.

Voilà ce qu'on peut donner en conclusion à ces quelques réflexions sur le dernier congrès de la FTQ. Cette centrale est en marche vers une certaine unité. La ligne de démarcation des deux anciennes centrales qui se sont fusionnées en 1956 tend à disparaître. Sur certaines questions, de nouvelles lignes se forment soit à cause de différences idéologiques, soit pour des raisons tactiques. Il reste à souhaiter que les divergences ne deviendront jamais fossés et que la FTQ pourra bénéficier pendant un certain temps d'une unité de pensée et d'action sur les questions primondiales.

\section{N'OU BLIEZ PAS}

Le Congrès des Relations industrielles de Laval

les 20 et 21 avril 1959

au Château Frontenac 